\title{
Experimental Research of Statistical Characteristics of Wind Wave Breaking
}

\author{
A. E. Korinenko ${ }^{1, *}$, V. V. Malinovsky ${ }^{1}$, V. N. Kudryavtsev ${ }^{1,2}$ \\ ${ }^{1}$ Marine Hydrophysical Institute, Russian Academy of Sciences, Sevastopol, Russian Federation \\ *e-mail: korinenko.alex@gmail.com \\ ${ }^{2}$ Russian State Hydrometeorological University, Saint-Petersburg, Russian Federation
}

The results of characteristics' analysis of the wind wave breaking (length, velocity, direction) obtained in the Black Sea in September-October, 2015 from the oceanographic platform in Katsiveli are presented. Wave breaking was recorded by a video camera synchronously with the measurements of wind waves and meteorological parameters. The algorithm based on calculating the threshold determined at analyzing the distribution function of the video signal brightness was applied to identify breaking using the video records. The optical equipment used in the experiment made it possible to reliably identify the breaking generated by the waves with the lengths greater than $4 \mathrm{~m}$ and the phase velocities exceeding $2.5 \mathrm{~m} / \mathrm{s}$. The data obtained correspond well to the model conceptions of O.M. Phillips which were developed for the equilibrium interval of the wind wave spectrum. The histograms of the wave breaking velocities at the wind speeds $10-16 \mathrm{~m} / \mathrm{s}$ are given. It is shown that at the developing waves, the breaking waves' phase velocity can reach that of the wind waves corresponding to the spectral peak, while at the developed waves, no breaks with the velocities exceeding a halfphase one of the waves corresponding to the spectral peak were observed. Probability densities of the breaking lengths in the wind speeds' measured range are described by the power law with the exponent -3.23. Distribution of the breaking orientations is described well by a degree of the angle cosine, at that the exponent decreases linearly from 5 to 4 with the wind speed increase from 10 to $16 \mathrm{~m} / \mathrm{s}$.

Keywords: wind wave breaking, in situ studies, wave breaking orientation, breaking lengths' distribution, wind waves' spectrum.

Acknowledgements: the investigation is carried out within the framework of the state task on the theme No. 0827-2018-0003 "Fundamental studies of the oceanological processes conditioning the marine environment state and evolution under the influence of natural and anthropogenic factors based on the observational and modeling methods". V.N. Kudryavtsev points to financial support of the RSF grant No. 17-77-30019.

For citation: Korinenko, A.E., Malinovsky, V.V. and Kudryavtsev, V.N., 2018. Experimental Research of Statistical Characteristics of Wind Wave Breaking. Physical Oceanography, [e-journal] 25(6), pp. 489-500. doi:10.22449/1573-160X-2018-6-489-500

DOI: $10.22449 / 1573-160 X-2018-6-489-500$

(C) 2018, A. E. Korinenko, V. V. Malinovsky, V. N. Kudryavtsev

(C) 2018, Physical Oceanography

\section{Introduction}

Wind wave breaking (WWB) plays an important role in the processes related to the gas exchange between the ocean and the atmosphere [1], wave energy dissipation [2], generation of turbulence in the near-surface sea layer [3].

The consideration of electromagnetic waves scattering at their breaking is important when analyzing the data of the ocean remote sensing (particularly, the radar data). Internal waves, areas of current convergence and other subsurface processes can cause an intensification of breaking and manifest themselves in the radar images of the sea $[4,5]$. 
The parameter used in different radiophysical tasks is the length of breaking crest $L_{\mathrm{b}}$. In [6], a significant role of the drops generated by WWB when scattering in the 8-millimeter range of electromagnetic waves is shown, and the intensity of the dropsis determined by $L_{\mathrm{b}}$ value. A model of nonresonant scattering component (related to WWB) at small grazing angles on the horizontal polarization was proposed in [7], where it was shown that the normalysed radar cross section depends on $L_{\mathrm{b}}$ and thewave breaking orientation.

A number of studies on the WWB lengths and orientations have been performed to date. In one of the first works [8] devoted to the study of breaking statistical characteristics, the probability densities of $L_{\mathrm{b}}$ values, well described by the gamma distribution, and the histograms of breaking orientations, described by the normal distribution, were obtained. The results of field experiment analysis are presented in [9], where $L_{\mathrm{b}}$ distribution is requested to be described by an exponential law. The histograms of breaking lengths and orientations are given in [10], and in [11] it is shown that at young waves the breaking rates can reach the phase velocity of the spectral peak waves, while at fully developed waves the ones of the spectrum equilibrium range break.

Breaking is the main source of wind-wave energy loss in the gravitational interval [2]. Therefore, the concepts of WWB angular distribution are required to validate the existing models of wave energy dissipation.

Despite a great number of works devoted to the study of breaking statistical characteristics, their comparisons with the model calculations are practically absent. The orientation of breaking lengths under various hydrometeorological conditions has not been sufficiently studied.

The purpose of this work is to analyze the statistical characteristics of windwave breaking obtained under field conditions, and to compare experimental dependencies with the existing models.

\section{The methods of data processing and experimental conditions}

Field experiments were carried out in September - October 2015 from a stationary oceanographic platform located in the Blue Bay near Katsiveli village (the Southern Coast of Crimea). The oceanographic platform is installed $\sim 480 \mathrm{~m}$ from the nearest point of the coast and has $44^{\circ} 23^{\prime} 38^{\prime \prime} \mathrm{N}, 33^{\circ} 59^{\prime} 09^{\prime \prime} \mathrm{E}$ coordinates. In the area of the platform the depth is about $30 \mathrm{~m}$.

In this work the determination of wind-wave breaking characteristics was carried out using video recordings of the sea surface made with a digital video camera located at $11.4 \mathrm{~m}$ height above sea level. The viewing direction was $30^{\circ}-40^{\circ}$ to the horizon, in the azimuthal plane - about $50^{\circ}-60^{\circ}$ to the "up-wind" direction. The use of a lens with $54^{\circ}$ horizontal and $32^{\circ}$ vertical viewing angle provided video recording of the region (area) on the sea surface in the form of a trapezium with 14-16 and 29-48 m base lengths. Video recording was carried out with 25 frames per second rate and $1920 \times 1080$ pixels resolution.

In order to identify the breakings against the sea surface, the original technique proposed in [12] was applied. Its algorithm is based on the threshold calculation, which is determined on the basis of the analysis of the distribution function of fullscale video recording brightness variations. The sea surface areas, the brightness of which was above the threshold value, were designated by a logical unit, all others 490

PHYSICAL OCEANOGRAPHY VOL. 25 ISS. 6 (2018) 
by zero. Subsequent data processing consisted in dividing the breaking process into two phases - the active crest and the passive foam. The selection of the active foamwas based on the differences in the kinematic characteristics of these phases [12]. Processing of videos was carried out automatically.

Further, the selected areas were divided into groups all points in which belong to areas related in space and time. As a result, a set of groups, each of which was information about the active phase evolution of the individual breaking, was ob-tained for all series of measurements.

In order to determine physical dimensions of breaking each video frame with a known observation geometry and camera parameters (tilt angle, matrix dimensions, focal length and lens viewing angles) was transformed into a rectangular system of horizontal coordinates on the sea surface. Depending on the angle of the video camera's lens field of view, the maximum spatial resolution made up $\sim 1 \mathrm{~cm}$, the minimum one $\sim 2.5 \mathrm{~cm}$. As a result, the breaking area $S_{i}^{j}$, its maximum linear dimensions ${ }^{j}$, which are the lengths of the major axes ellipses approximating the breaking region according to the method [12], were calculated for each group. Along with the above mentioned parameters, the coordinates of mass center position $\left(x_{i}^{j}, y_{i}^{j}\right)$, where index $i$ is the number of breaking belonging to the group and $j$ is the number of a group were found. Average modulus of breaking motion velocity and propagation direction were calculated as follows: $c^{j}=\sqrt{\left(c_{x}^{j}\right)^{2}+\left(c_{y}^{j}\right)^{2}}$, $\phi^{j}=\arctan \left(c_{y}^{j} / c_{x}^{j}\right)$, where $c_{x}^{j}, c_{y}^{j}$ are the velocity components which were determined by the mass center motion using the method of the least-squares error method.

The result of processing is a database containing the information about linear dimensions of the breaking in time and space, average modulus of mass center motion velocity of breaking crest and its direction.

Meteorological information was collected by Davis 6152EU multifunctional complex located at $23 \mathrm{~m}$ height above the sea level on the mast of the oceanographic platform. The measured wind velocity was recalculated into effective neutral stratified wind velocity $U$ at $10 \mathrm{~m}$ height according to the method [13].

Surface wave characteristics were recorded using a grid of 6 string wave recorders located in the center and tops of a regular pentagon with $0.25 \mathrm{~m}$ circumra-dius. The distance of wave recorder grid to the nearest platform element exceeded $10 \mathrm{~m}$.

As a result of wave recorder data processing, we used the maximum entropy method to calculate frequency-angular spectra of the sea surface elevations $S(f, \phi)$, to determine the frequencies of wind waves spectral peak $f_{p}$, directions of general propagation of wind waves $\phi_{p}$ and swell waves, the significant wave heights $H_{S}\left(H_{S}=4 \sqrt{\sigma^{2}}\right.$, where $\sigma^{2}$ is the dispersion of sea surface elevations).

For the analysis of the obtained results,below we use the theory of O. M. Phillips [14], developed for the equilibrium interval of wind wave spectrum, where the energy inputfrom the wind, nonlinear interactions and energy dissipation due to breaking are in balance and are proportional to each other. 
As is known, in natural conditions, in addition to the wind waves, there are swell waves the evolution of which is not described within the local energy balance characteristic of the equilibrium interval.

In order to divide the frequency spectrum of waves into swell waves and wind ones, we used the method proposed in [15], in which the measured spectrum of $S(f)$ waves was compared with the theoretical spectrum $F(f)[14]: F(f)=$ $2 \pi \gamma u_{*} g(2 \pi f)^{-4}$, where $\gamma=0.06-0.11 ; u_{*}$ is dynamic velocity; $g$ is the gravitational acceleration. According to this method, the spectrum area which lies above the lower boundary $(\gamma=0.06)$ belongs to wind waves, and if the spectrum area is situated below the boundary, then the measured waves are classified as swell ones.

Our measurements were carried out within 10-16 m/s range of wind velocities. The main criterion for the measurement data selection was the stationarity of wind velocity and direction over several hours. For the analysis we selected the cases when the angle between the directions of swell waves and wind velocity did not exceed $30^{\circ}$.

General information on the experimental conditions is given in the table (measurement date; average wind velocity $\bar{U}$ and its direction $\varphi_{\bar{U}}$; $H_{s}$; wave age $\alpha=c_{p} / U$, where $c_{p}$ is the phase velocity of the waves at the frequency of spectral peak $f_{p}$ ).

Measuring conditions

\begin{tabular}{|c|c|c|c|c|c|c|}
\hline Date & $\begin{array}{l}\text { No of meas- } \\
\text { urement series }\end{array}$ & $\bar{U}, \mathrm{~m} / \mathrm{s}$ & $\varphi_{\bar{U}^{\circ}}^{\circ}$ & $H_{s}, \mathrm{~m}$ & $\alpha$ & $f_{p}^{\circ}$ \\
\hline \multirow{3}{*}{10.09 .2015} & 1 & 11.8 & 85 & 1.1 & 0.6 & 0.21 \\
\hline & 2 & 11.7 & 85 & 1.1 & 0.6 & 0.21 \\
\hline & 3 & 10.9 & 85 & 1.2 & 0.8 & 0.19 \\
\hline \multirow{3}{*}{11.09 .2015} & 1 & 10.0 & 90 & 0.8 & 0.6 & 0.28 \\
\hline & 2 & 10.3 & 90 & 0.8 & 0.6 & 0.26 \\
\hline & 3 & 11.0 & 90 & 0.8 & 0.6 & 0.26 \\
\hline \multirow{3}{*}{12.09 .2015} & 1 & 14.6 & 70 & 1.2 & 0.4 & 0.28 \\
\hline & 2 & 16.1 & 75 & 1.2 & 0.3 & 0.29 \\
\hline & 3 & 15.2 & 85 & 1.4 & 0.4 & 0.25 \\
\hline \multirow{3}{*}{08.10 .2015} & 1 & 13.2 & 70 & 1.3 & 0.4 & 0.29 \\
\hline & 2 & 13.4 & 80 & 1.5 & 0.5 & 0.24 \\
\hline & 3 & 11.7 & 80 & 1.5 & 0.6 & 0.24 \\
\hline 11.10 .2015 & 1 & 13.6 & 70 & 2.2 & 0.8 & 0.14 \\
\hline \multirow{2}{*}{12.10 .2015} & 1 & 14.4 & 65 & 2.2 & 0.8 & 0.13 \\
\hline & 2 & 15.0 & 65 & 2.0 & 0.7 & 0.14 \\
\hline
\end{tabular}

\section{The results of field measurements}

Distribution of wave breaking velocities. We consider the statistical characteristics of rate moduli $c$ of wind wave breakings. According to the assumption [14], the rate of breaking motion is equal to the phase velocity $c$ of breaking wave. In this case, the analysis of breaking histograms depending on $c$ provides, taking 
into account the dispersion relation, the estimation of probability of wind wave breaking of various lengths.

The distributions of breaking rates per surface unit $n_{\mathrm{br}}(c)$, obtained from each videotape, are shown in Fig. 1, a. In the presented data, at $c>2.5-3 \mathrm{~m} / \mathrm{s}$ a sharp drop in $n_{\mathrm{br}}(c)$ is observed with increasing breaking rates. When $c<\sim 2.5 \mathrm{~m} / \mathrm{s}$ the slope of histograms is significantly smaller and in a number of records $n_{\mathrm{br}}(c)$ values are almost constant in this interval. Such feature of the histograms of breaking characteristics is explained in detail in [16]. This is due to the fact that when using optical equipment a large number of breakings formed by short waves are not identified during the video processing.
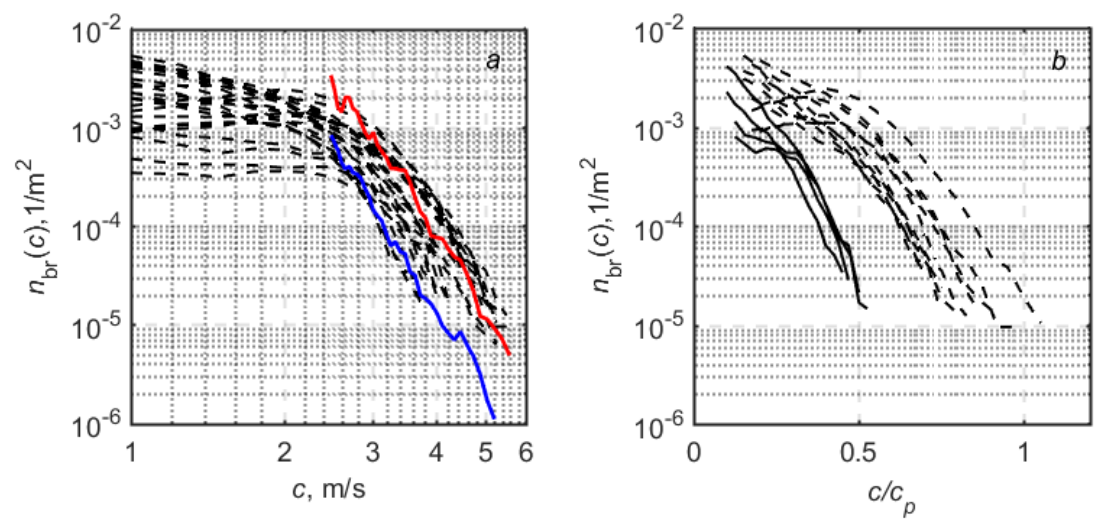

F i g. 1. Statistical characteristics of wave breaking rates: $a$ - histograms of wave breaking velocities at the wind velocities 10-16 m/s (solid lines are the results of calculations by formula (9) (see p. 496) for the wind velocity $10 \mathrm{~m} / \mathrm{s}$ (blue curve) and $16 \mathrm{~m} / \mathrm{s}$ (red curve)); $b$ - histograms of the relation of wave breakingrate to the phase velocity corresponding to the spectral peak in the wave age range 0.3-0.5 (dash line) and 0.65-0.8 (solid line)

Fig. $1, b$ shows $n_{\mathrm{br}}(c)$, where the values of $c / c_{p}$ ratios characterizing the spectral interval of breaking wave are indicated on the abscissa axis. With developing waves, the phase velocity of the breaking waves reaches the phase velocity of the wind waves of the spectral peak. With the development of waves, we did not observe breakings moving at the velocities above $0.5 c_{p}$. The obtained result correlates with the experimental data $[11,17]$.

Distribution of breaking lengths. We consider statistic characteristics of $L_{\mathbf{b}}$ breaking lengths determined as the foam line size in the direction parallel to the crest of breaking wave. In Fig. 2 the points show the distribution $p\left(L_{\mathrm{b}}\right)$ obtained in each videotape at different hydrometeorological conditions. Despite a rather wide range of measurement conditions, all distributions are rather similar.

As follows from Fig. 2, a, the densities of breaking length probabilities are generally close to the power law

$$
p\left(L_{\mathrm{b}}\right)=\mu L_{\mathrm{b}}^{-\rho} .
$$

The solid line shows the calculation by the formula (1), where the coefficients for the set of data for all records were found by the method of least-squares errors method and made up $\mu=0.38$ and $\rho=3.23$. 

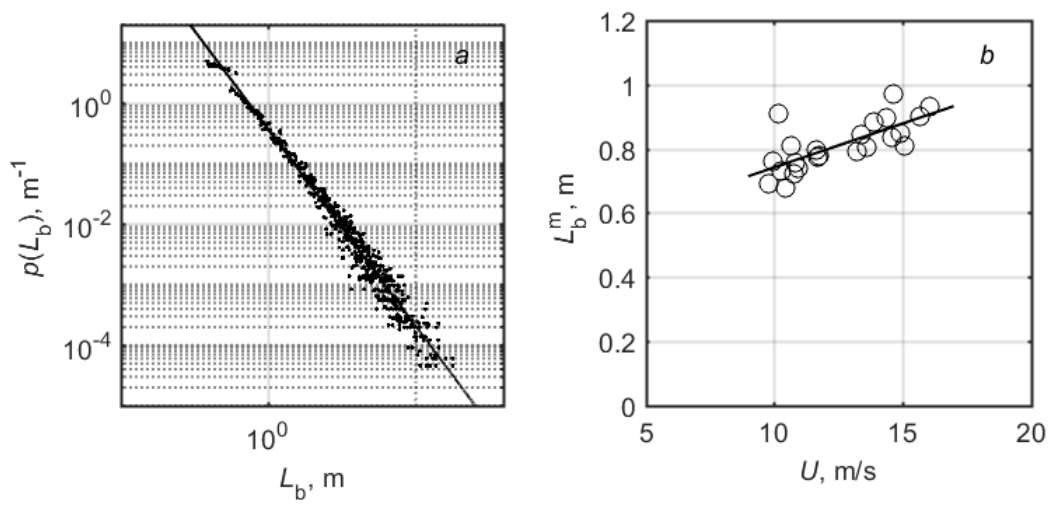

F i g. 2. Statistical characteristics of the wave breaking lengths resulted from all the records: $a$ - distribution of $L_{\mathrm{b}}$ values; $b$ - wind dependence of the wave breaking average length (circles), solid line denotes data approximation by linear dependence

From Fig. 2, $b$ it follows that with $U$ increase from 10 to $16 \mathrm{~m} / \mathrm{s}$ average values of $L \mathrm{~b}$ increase from $\sim 0.7$ to $\sim 0.9 \mathrm{~m}$. Straight $l$ ine shows linear dependence $L$ $={ }_{b}^{m} 0.47+0.027 U$ the inclination of which is close to 0.034 value obtained in [8]. Weak wind dependence of $L$ malues can be explained by sharp decrease of $p\left(L_{\mathrm{b}}\right)$ with the growth of WWB length. Indeed, the average length of the breaking major axis is equal to the first moment of random variable $L_{b}$. Then, taking into account (1) and the obtained inclination $\rho$, we can estimate $L_{\mathrm{b}}^{\mathrm{m}} \sim\left(\mathrm{L}_{\mathrm{b}}^{\min }\right)^{-2.23}-$ $\left(\mathrm{L}_{b}{ }^{\mathrm{max}}\right)^{-2.23}$, where $\mathrm{L}_{\mathrm{b}}{ }^{\min }$ and ${ }_{h}{ }^{\max }$ are the minimum and the maximum values of registered $L_{b}$. Thus, $L_{\mathrm{b}}^{m}$ is mainly determined by its minimum size $\mathrm{L}_{\mathrm{b}}^{\min }$ (specified when analyzing the data) and increases with $L_{\mathrm{b}}^{\max }$ growth and increase of the wind.

Distribution of breaking orientation. In Fig. 3 the example of angular distribution histograms of breaking number obtained on September 10 and 12, 2015 during the second series of measurements, is shown. Solid line indicates the approximation by the function

$$
P_{n}(\phi)=A \cos ^{p}(\phi)
$$
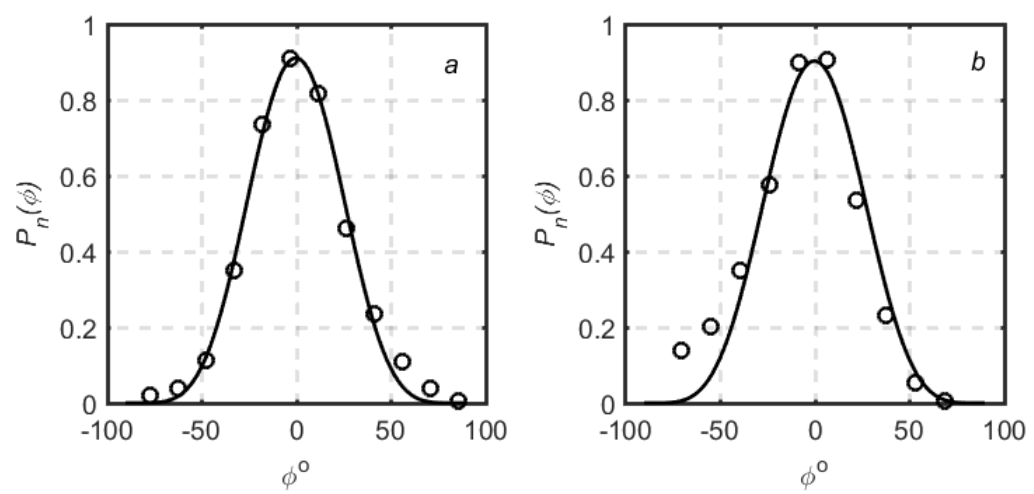

F i g. 3. Distribution of wave breaking orientations obtained on 10.09.2015 - $a$ and 12.09.2015 - $b$ 
For convenience of comparison, all angular distributions were normalized as follows: $\int_{-\pi / 2}^{\pi / 2} P_{n}(\phi) d \phi=1$, where $P_{n}(\phi)=A n_{\mathrm{br}}(\phi), A^{-1}=\int_{-\pi / 2}^{\pi / 2} n_{\mathrm{br}}(\phi) d \phi$, $n_{\mathrm{br}}(\phi)$ is a number of breakings in the direction $\phi$. The origin of coordinates corresponds to the maximum of distribution. It can be seen that the distributions of orientations have symmetric unimodal form and are well described by the expres$\operatorname{sion}(2)$.

The dependence of $p$ values (obtained for all data) on wind velocity is shown in Fig. 4, $a$ where rather significant scattering of points is observed. Nevertheless, a weak wind dependence of the exponent is observed, which can be approximated by a linear function $p=6.5-0.15 U$ (solid line). The $p$ values obtained at various wave ages are shown in Fig. 4, $b$, where the absence of an explicit dependence of the exponent on $\alpha$ is obvious.
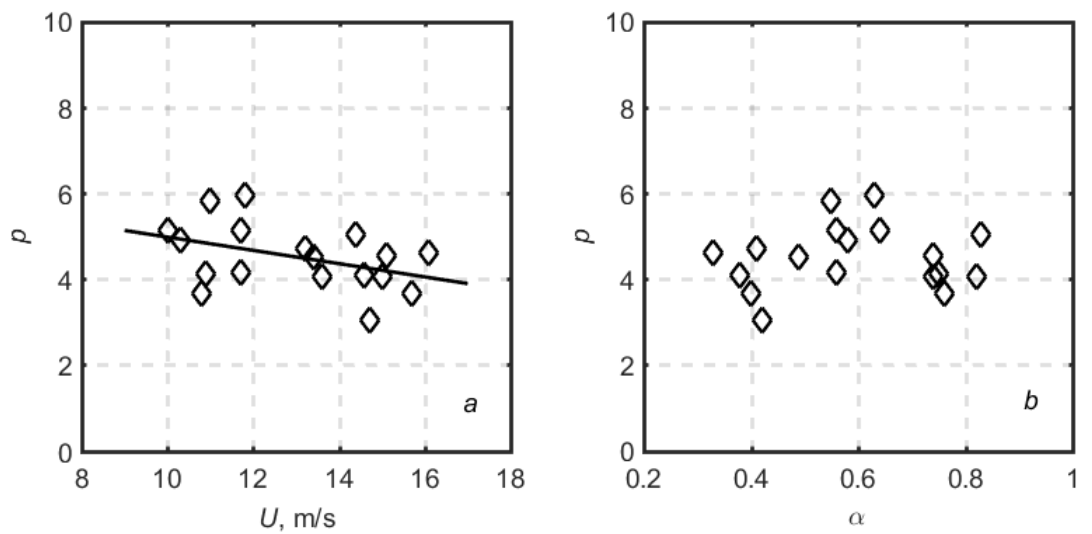

F i g. 4. Dependence of exponent $p$ (formula (2)) on the wind velocity- $a$ and wave age $-b$

\section{Discussion of the results}

The breakings visualize energy dissipation of wind waves. It is believed that breaking is the main mechanism responsible for the discharge of gravitational waves energy in the equilibrium range of the spectrum. The distribution of breaking waves by the direction of motion is an important characteristic that allows us to relate spatial characteristics of breaking to the angular spectrum of the surface waves.

In order to assess the statistical characteristics of wind-wave breaking, we use the breaking statistics proposed by O. M. Phillips [14]: the author describes dynamic, kinematic and statistical properties of the WWB through the length of the crest of the breaking wave moving at $\mathbf{c}$ velocity. $\Lambda(\mathbf{c})$-function w as proposed -the distribution of the total length of breaking crests per surface unit within the range of velocities $(\mathbf{c}, \mathbf{c}+d \mathbf{c})$. The integral $\int \Lambda(\mathbf{c}) d \mathbf{c}$ is the total length of the breaking crests per unit of the sea surface area.

Assuming that the WWB are geometrically similar, the average length of breaking crests will be proportional to $k^{-1}$, where $k$ is a wave number of breaking wave. Then the number of $n_{\mathrm{br}}$ breakings per unit of the area will be proportional to:

$$
n_{\mathrm{br}} \sim k \Lambda(\mathbf{k}) d \mathbf{k},
$$


where $\Lambda(\mathbf{k})$ is total length of breaking crests per surface unit within the range of wave numbers ( $\mathbf{k}, \mathbf{k}+d \mathbf{k})$. It should be pointed out that in the transition from $\Lambda(\mathbf{c})$ to $\Lambda(\mathbf{k})$ the dispersion relation for gravitational waves in deep water $\omega^{2}$ $=$ and the expression for phase velocity $c^{2}=g / k$ are used.

An important conclusion of O. M. Phillips theory is the connection of $\Lambda$-function with the average rate of energy dissipation $D(\mathbf{k})$ on the unit of surface due to breakings [14]:

$$
D(\mathbf{k})=b g^{3 / 2} k^{-5 / 2} \Lambda(\mathbf{k}),
$$

where $b=0.03-0.07$ is dimensionless parameter [18].

In order to find $D(\mathbf{k})$ we consider the energy balance in equilibrium interval of surface wave spectrum. According to [14], energy dissipation due to breakings will be proportional to the energy input from the wind:

$$
D(\mathbf{k})=g \omega \beta(k, \phi) F(\mathbf{k}),
$$

where $\beta(k, \phi)$ is a coefficient of wind-wave interaction; $F(\mathbf{k})$ is a spectrum of elevations.

From (4) and (5) we can obtain the expression $\Lambda(\mathbf{k})=b^{-1} k^{3} \beta(k, \phi) F(\mathbf{k})$, substituting which in (3) we write down $n_{\mathrm{br}}$ in the following form:

$$
n_{\mathrm{br}}(\mathbf{k}) \sim b^{-1} \beta(k, \phi) k^{4} F(\mathbf{k}) .
$$

In [19] an expression for wind-wave interaction $\beta(k, \phi)=C_{\beta}\left(u_{*} / c\right)^{2} \cos ^{2} \phi$, where $C_{\beta}$ is a parameter, is proposed. In the variables $(\omega, \phi)$, taking into account the dispersion relation, the expression (6) can be written as

$$
n_{\mathrm{br}}(\omega, \phi) \sim b^{-1} C_{\beta} u_{*}^{2} g^{-6} \cos ^{2} \phi \omega^{10} S(\omega, \phi) .
$$

According to (7), the azimuthal distribution of number of breakings generated by the waves from frequency interval $\left(\omega_{0}, \omega_{1}\right)$ will be written as

$$
n_{\mathrm{br}}(\phi) \sim b^{-1} C_{\beta} u_{*}^{2} g^{-6} \int_{\omega_{0}}^{\omega_{1}} \cos ^{2} \phi \omega^{10} S(\omega, \phi) d \omega,
$$

where $\omega_{0}=1.5 \omega_{p}$ and $\omega_{1}$ are the upper and the lower boundaries of equilibrium interval, respectively [20]. As it was mentioned above, the applied method provides a reliable recording of breakings the velocity of which exceeds $2.5 \mathrm{~m} / \mathrm{s}$. Then, for our conditions $\omega_{1}=4 \mathrm{rad} / \mathrm{s}$.

Expression (7) allows one to estimate the number of breakings on the surface unit within the range of velocities $(c, c+d c)$. Integrating (7) over the azimuth, taking into account the dispersion relation in the variables $c$, we can write down

$$
n_{\mathrm{br}}(c) \sim b^{-1} C_{\beta} u_{*}^{2} g^{2} c^{-7} \int_{-\frac{\pi}{2}}^{\frac{\pi}{2}} \cos ^{2} \phi B(\omega, \phi) d \phi,
$$

where $B(\omega)=S(\omega, \phi) \omega^{5} / 2 g^{2}$ is a function of saturation [14].

Total number of breakings $n_{\mathrm{br}}$ (moving at the velocities within the range $c_{0}-c_{1}$ ) on the unit of area at this point in time will be written as

$$
n_{\mathrm{br}}\left(c_{0}, c_{1}\right) \sim b^{-1} C_{\beta} u_{*}^{2} g^{2} \int_{c_{1}}^{c_{0}} \int_{-\frac{\pi}{2}}^{\frac{\pi}{2}} c^{-7} \cos ^{2} \phi B(\omega, \phi) d \phi d c,
$$


where $c_{0}=0.7 c_{p} ; c_{1}=2.5 \mathrm{~m} / \mathrm{s}$ is the minimum breaking rate reliably recorded by the applied method for processing the video images.

For a quantitative comparison of the experimentally measured statistical characteristics of wind wave breaking with the model expressions (8) - (10), it is necessary to determine the values of the constant $b$ and the proportionality coefficient connecting the average length of the breaking crest with the wavenumber. As follows from [21], the values $b$, obtained by different authors, differ more than by 3 orders of magnitude. As the assessment of constants is beyond the scope of this work, we will conduct a qualitative comparison of measurement results and model calculations (8) - (10). For $S(\omega, \phi)$ we take the frequency-angle spectra of sea surface elevations measured during the experiments.

In Fig. 5 the examples of distributions of breaking orientations are shown: dashed lines - the data obtained from sea surface video recordings, solid lines - the data calculated from the model (6) with regard to measured wave spectra.
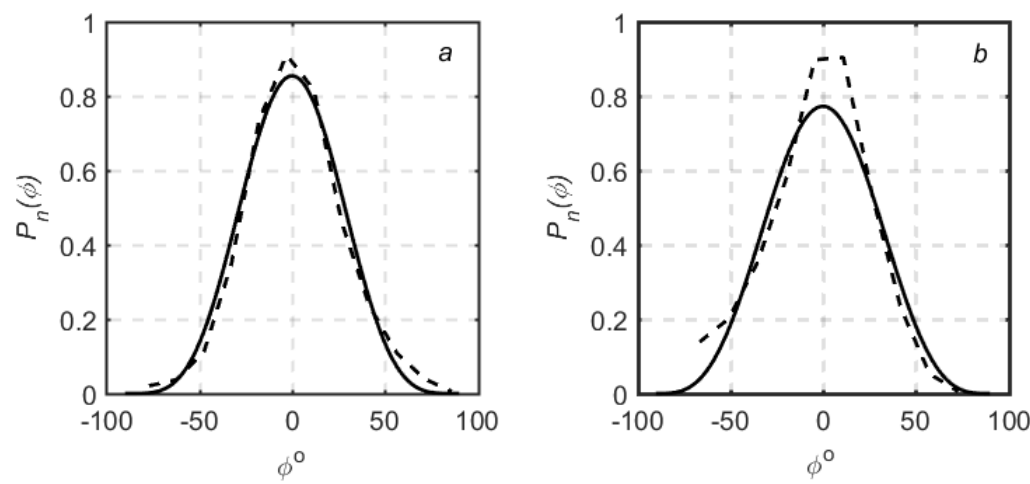

F i g. 5. Distribution of wave breaking quantity according to directions: $a$ - at wind velocity $11.7 \mathrm{~m} / \mathrm{s}$ and wave age $0.64 ; b-$ at $U=16 \mathrm{~m} / \mathrm{s}$ and wave age 0.33

In order to compare the distributions $P_{n}(\phi)$ obtained from experimental data and model calculations, we approximate the angular distributions of breaking number and expression (8) by a power cosine function with exponent $p$. A comparison of values of $p_{\mathrm{br}}$ coefficients found in the approximation of field data and the values of $p_{\mathrm{wg}}$ obtained in the approximation (8), is shown in Fig. 6, where there is a satisfactory correlation of experimental results and model calculations. However, series of $p_{\mathrm{wg}}$ values is approximately 1.2-1.5 times less than the corresponding $p_{\mathrm{br}}$ values. Such discrepancy in the width of breaking orientation distributions requires additional study. One of the causes may be the modulating effect of long waves, which is not taken into account in the model.

As it was mentioned above, $b$ value requires further adjustment since it differs by several orders of magnitude in the works of different authors. In this case, we can only compare the type of model dependencies with the results of field measurements. In calculations based on (9), (10), the values of the constants were selected so that the levels of the model and experimental dependences were close. In Fig. 1, a colored lines show the breaking rate distributions calculated from (9), and the measured frequency-angular spectra of sea surface elevations are used as $S(\omega, \phi)$. 
As follows from Fig. 1, $a$, the inclinations of the curves calculated from (9) are in good agreement with the field data. We note that the levels of model distributions at a fixed breaking rate grow with the wind increase, which explains the observed variation in the experimental values of $n_{\mathrm{br}}(c)$ obtained at different winds.
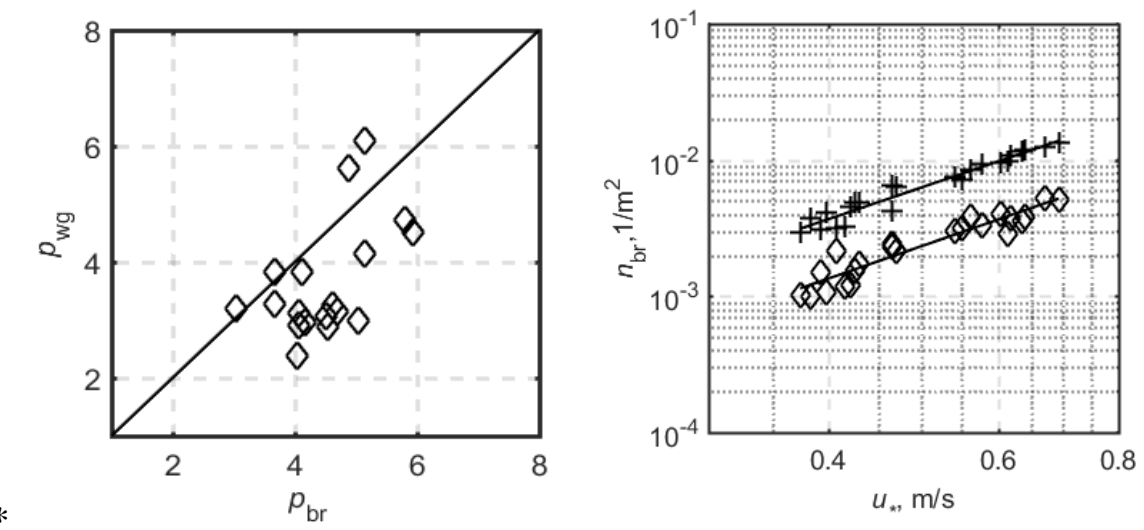

F i g. 6. Exponent of function $\cos ^{p}(\phi \quad)$ at approximation of in situ data and expression (8). Solid line corresponds to equality of two values

F i g. 7. Dependence of total wave breakings per unit of surface $(c>2.5 \mathrm{~m} / \mathrm{s})$ on the dynamic velocity

We consider the behavior of the total number of breakings $n_{\mathrm{br}}$ obtained in various conditions. In Fig. 7 rhombuses show the experimental dependence $n_{\text {br }}$, crosses - the results of calculations according to (10), straight lines - approximation by the dependence $n_{\mathrm{br}}=0.0128 u_{*}^{2,5}$ (for rhombuses) and $n_{\mathrm{br}} \sim u_{*}^{2.4}$ (for crosses), where the coefficients are calculated by the method of the least-squares errors. It can be seen that the inclinations of the model and experimental wind dependences $n_{\mathrm{br}}$ coincide.

\section{Conclusion}

The performed analysis of the statistical characteristics of wind wave breaking allows us to draw the following conclusions. Depending on the sea surface condition, breaking is formed in different intervals of the wave spectrum. At the developing sea, the waves of equilibrium interval with phase velocities not exceeding 0.5 of the one of spectral peak break. At a developing sea, the velocities of breaking waves reach the phase velocity of the wind waves of spectral peak. The distribu-tions of breaking lengths are described well by a power law with -3.23 exponent. Average WWB lengths have a weak linear growth with wind velocity increase.

Distributions of breaking orientations (moving at the velocities within the interval $c_{0}-c_{1}$, where $\left.c_{0}=0.7 c_{p} ; c_{1}=2.5 \mathrm{~m} / \mathrm{s}\right)$ can be approximated by $A \cos ^{p}(\phi)$ function. The values of exponent $p$ linearly decrease from 5 to 4 with wind velocity increase from 10 to $16 \mathrm{~m} / \mathrm{s}$. No explicit dependence of $p$ values on the age of the waves was found.

In order to assess the statistical characteristics of wind wave breaking obtained in field conditions, an approach of O. M. Phillips, relating dynamic, kinematic and statistic properties of WWB with the lengths of breaking wave crests ( $\Lambda$-function) was used. Taking into account this approach, $\Lambda$-function can be expressed through 
the wind-wave interaction coefficient $\beta(\omega, \phi)$ and wave spectrum $S(\omega, \phi)$ and thus construct a model for the number of breaking per unit of surface. The correctness of the applied theoretical approach to the description of breaking statistic characteristics is confirmed by comparing experimental data with model calculations.

Comparison of model and field distributions of WWB orientations at their approximation by $A \cos ^{p}(\phi)$ function showed their good correlation. At the same time, for a number of measurements the calculated values $p$ turned out to be approximately 1.2-1.5 times less than those obtained from experimental data.

Inclinations if breaking rate distributions at $c>2.5 \mathrm{~m} / \mathrm{s}$, obtained during the measurements and as a result of model calculations, coincide. At the same time, the model demonstrates the growth of $n_{\mathrm{br}}(c)$ level with the wind increase. This explains the scattering of the observed data. Wind dependence of breaking number per unit of area at $c>2.5 \mathrm{~m} / \mathrm{s}$ is described well by power law $n_{\mathrm{br}}=0.0128 u_{*}^{2,5}$. Inclination of model curve $n_{\mathrm{br}} \sim u_{*}^{2,4}$ coincides with the field data.

\section{REFERENCES}

1. Zappa, C.J., McGillis, W.R., Raymond, P.A., Edson, J.B., Hintsa, E.J., Zemmelink, H.J., Dacey, J.W.H. and Ho, D.T., 2007. Environmental Turbulent Mixing Controls on Air-Water Gas Exchange in Marine and Aquatic Systems. Journal of Geophysical Research Letters, [ejournal] 34(10), L10601. https://doi.org/10.1029/2006GL028790

2. Thorpe, S.A., 1993. Energy Loss by Breaking Waves. Journal of Physical Oceanography, [e-journal] 23(11), pp. 2498-2502. https://doi.org/10.1175/1520-0485(1993)023<2498:ELBBW>2.0.CO;2

3. Kudryavtsev, V., Shrira, V., Dulov, V. and Malinovsky, V., 2008. On the Vertical Structure of Wind-Driven Sea Currents. Journal of Physical Oceanography, [e-journal] 38(10), pp. 2121-2144. https://doi.org/10.1175/2008JPO3883.1

4. Johannessen, J.A., Kudryavtsev, V., Akimov, D., Eldevik, T., Winther, N. and Chapron, B., 2005. On Radar Imaging of Current Features: 2. Mesoscale Eddy and Current Front Detection. JGR: Oceans, [e-journal] 110(C7), C07017. https://doi.org/10.1029/2004JC002802

5. Mouche, A.A., Hauser, D. and Kudryavtsev, V., 2006. Radar Scattering of the Ocean Surface and Sea-Roughness Properties: A Combined Analysis from Dual-Polarizations Airborne Radar Observations and Models in C Band. JGR: Oceans, [e-journal] 111(C9), C09004. https://doi.org/10.1029/2005JC003166

6. Yurovsky, Y.Y. and Malinovsky, V.V., 2012. Radar Backscattering from Breaking Wind Waves: Field Observation and Modelling. International Journal of Remote Sensing, [e-journal] 33(8), pp. 2462-2481. https://doi.org/10.1080/01431161.2011.614966

7. Churyumov, A.N. and Kravtsov, Yu.A., 2000. Microwave Backscatter from Mesoscale Breaking Waves on the Sea Surface. Waves in Random Media, [e-journal] 10(1), pp. 1-15. https://doi.org/10.1088/0959-7174/10/1/301

8. Bondur, V.G. and Sharkov, E.A., 1990. Statistical Characteristics of Linear Geometric Elements of Foam Structures on the Sea Surface for Optical Sensor Data. Soviet Journal of Remote Sensing, [e-journal] 6(4), pp. 534-550.

9. Mironov, A.S. and Dulov, V.A., 2008. Statisticheskie Kharakteristiki Sobytiy i Dissipatsiya Energiipri Obrushenii Vetrovykh Voln [Statistical Properties of Individual Events and Energy Dissipation of Breaking Waves]. In: MHI, 2008. Ekologicheskaya Bezopasnost' Pribrezhnoy $i$ Shel'fovoy Zoni Kompleksnoe Ispol'zovanie Resursov Shel'fa [Ecological Safety of Coastal and Shelf Zones and Comprehensive Use of Shelf Resources]. Sevastopol: MHI. Iss. 16, pp. 97-115 (in Russian).

10. Gemmrich, J., Zappa, C.J., Banner, M.L. and Morison, R.P., 2013. Wave Breaking in Developing and Mature Seas.JGR: Oceans, [e-journal] 118(9), pp. 4542-4552. https://doi.org/10.1002/jgrc.20334

11. Gemmrich, J.R, Banner, M.L. and Garrett, C., 2008. Spectrally Resolved Energy Dissipation Rate and Momentum Flux of Breaking Waves. Journal of Physical Oceanography, [e-journal] 38(6), pp. 1296-1312. https://doi.org/10.1175/2007JPO3762.1 
12. Mironov, A.S. and Dulov, V.A., 2007. Detection of Wave Breaking Using Sea Surface Video Records. Measurement Science and Technology, [e-journal] 19(1), 015405. doi:10.1088/0957-0233/19/1/015405

13. Fairall, C.W., Bradley, E.F., Hare, J.E., Grachev, A.A. and Edson, J.B., 2003. Bulk Parameterization of Air-Sea Fuxes: Updates and Verification for the COARE Algorithm. Journal of Climate, [e-journal] 16(4), pp. 571-591. https://doi.org/10.1175/1520-0442(2003)016<0571:BPOASF>2.0.CO;2

14. Phillips, O.M., 1985. Spectral and Statistical Properties of the Equilibrium Range in WindGenerated Gravity Waves. Journal of Fluid Mechanics, [e-journal] 156, pp. 505-531. https://doi.org/10.1017/S0022112085002221

15. Hanson, J.L. and Phillips, O.M., 1999. Wind Sea Growth and Dissipation in the Open Ocean. Journal of Physical Oceanography, [e-journal] 29(8), pp. 1633-1648. https://doi.org/10.1175/1520-0485(1999)029<1633:WSGADI>2.0.CO;2

16. Sutherland, P. and Melville, W.K., 2013. Field Measurements and Scaling of Ocean Surface Wave-Breaking Statistics. Geophysical Research Letters, [e-journal] 40(12), pp. 3074-3079. https://doi.org/10.1002/grl.50584

17. Kleiss, J.M. and Melville, W.K., 2010. Observations of Wave Breaking Kinematics in FetchLimited Seas. Journal of Physical Oceanography, [e-journal] 40(12), pp. 2575-2604. https://doi.org/10.1175/2010JPO4383.1

18. Duncan, J.H. and Longuet-Higgins, M.S., 1981. An Experimental Investigation of Breaking Waves Produced by a Towed Hydrofoil. Proceedings of the Royal Society of London. A. Mathematical and Physical Sciences, [e-journal] 377(1770), pp. 331-348. https://doi.org/10.1098/rspa.1981.0127

19. Makin, V.K. and Kudryavtsev, V.N., 1999. Coupled Sea Surface-Atmosphere Model: 1. Wind Over Waves Coupling. JGR: Oceans, [e-journal] 104(C4), pp. 7613-7623. https://doi.org/10.1029/1999JC900006

20. Donelan, M.A., Hamilton, J., Hui, W.H. and Stewart, R.W., 1985. Directional Spectra of Wind-Generated Ocean Waves. Philosophical Transactions of the Royal Society of London. Series A, Mathematical and Physical Sciences, [e-journal] 315(1534), pp. 509-562. https://doi.org/10.1098/rsta.1985.0054

21. Romero, L., Melville, W.K. and Kleiss, J.M., 2012. Spectral Energy Dissipation due to Surface Wave Breaking. Journal of Physical Oceanography, [e-journal] 42(9), pp. 1421-1444. https://doi.org/10.1175/JPO-D-11-072.1

About the authors:

Aleksandr E. Korinenko - Research Associate, Remote Sensing Department, FSBSI MHI (2 Kapitanskaya St., Sevastopol, 299011, Russian Federation), Ph.D. (Phys.-Math.), Scopus Author ID: 23492523000, korinenko.alex@gmail.com

Vladimir V. Malinovsky - Senior Research Associate, Remote Sensing Department, FSBSI MHI (2 Kapitanskaya St., Sevastopol, 299011, Russian Federation), Ph.D. (Phys.-Math.), Scopus Author ID: 23012976200, dvs-ltd@mail.ru

Vladimir N. Kudryavtsev - Leading Research Associate, Remote Sensing Department, FSBSI MHI (2 Kapitanskaya St., Sevastopol, 299011, Russian Federation), Dr.Sci. (Phys.-Math.); Executive Director of the Laboratory of Satellite Oceanography, Russian State Hydrometeorological University (79 Voronezhskaya St., Saint Petersburg, 192007, Russian Federation), Scopus Author ID: 7102703183,kudr@rshu.ru

Contribution of the co-authors:

Aleksandr E. Korinenko - development of techniques and carrying out the experimental studies, analysis and synthesis of the research results, preparation of the text of the article.

Vladimir V. Malinovsky - development of experimental research methods, analysis and synthesis of research results, participation in the discussion of the article materials, preparation of the article text.

Vladimir N. Kudryavtsev - general scientific management of the research, description of the research results, refinement of the text of the article.

All the authors have read and approved the final manuscript.

The authors declare that they have no conflict of interest. 